\title{
Another possibility of sonoluminescence due to the cherenkov radiation from the ZPF field in a water bubble
}

\author{
Takaaki Musha
}

Advanced Sci.-Tech. Research. Organization, Namiki, Kanazawa-ku, Yokohama, Japan; takaaki.musha@gmail.com

Received 13 January 2011; revised 10 February 2011; accepted 15 February 2011.

\begin{abstract}
Sonoluminescence is the light produced from the collapse of bubbles in water under ultrasound. Schwinger proposed a physical mechanism for sonoluminescence in terms of photon production due to changes of quantum electrodynamic energy contained in a collapsing dielectric bubble. However there are critics for the Schwinger's proposal that his estimate of the Casimir energy involved is inaccurate and there are several papers to propose its missing term. In this paper, the author presents another possible component of sonoluminescense which is due to Cherenkov radiation from tachyon pairs generated in a collapsing bubble.
\end{abstract}

Keywords: Sonoluminscence; Casimir Energy; Cherenkov Radiation; Tachyon; Zero-Point Energy

\section{INTRODUCTION}

Cavitation is the formation of vapor bubbles of a flowing liquid in a region where the pressure of the liquid falls below its vapor pressure. This is a process in which a void or a bubble in a liquid rapidly collapses and producing a shock wave to cause a temperature increase and emits light. This phenomenon is now referred to as sonoluminescence (SL). Sonoluminescence can occur when a sound wave of sufficient intensity induces a gaseous cavity within a liquid to collapse quickly. The bubble reaches a maximum at about ten times its original size, it completely collapses within a few milliseconds later. Sonoluminescence observed in the laboratory can be made to be stable, so that a single bubble will expand and collapse over and over again in a periodic fashion, emitting a burst of light each time it collapses. For this to occur, a gas filled bubble undergoes repeated growth and collapse in response to an acoustic standing wave. As the radius of the bubble quickly shrinks, the potential energy is released as heat and light [1]. The experimental results obtained in various fluids have shown that; 1) a flash of light emitted from the collapsing bubble with a maximum diameter of 100 microns creates a temperature of $5500^{\circ} \mathrm{C}$ with a blackbody spectrum when it rapidly shrinks to less than one micron in radius, 2) light flashes from the bubbles are extremely short persisted only for 50 picoseconds or shorter with peak intensities of the order of $1 \sim 10 \mathrm{~mW}$, which was too brief for the light to be produced by some atomic process, 3) bubbles are very small when they emit the light with about $1 \mu \mathrm{m}$ in diameter depending on the ambient fluid and the gas content of the bubble, 4) single-bubble sonoluminescence pulses have very stable periods and positions and the frequency of light flashes can be more stable than the rated frequency stability of the oscillator making the sound waves driving them, and 5) the addition of a small amount of noble gas to the gas in the bubble increases the intensity of the emitted light. Spectral measurements have given bubble temperatures in the range from $2300^{\circ} \mathrm{K}$ to $5100^{\circ} \mathrm{K}$, the exact temperatures depending on experimental conditions including the composition of the liquid and gas [2]. The mechanism of the phenomenon of sonoluminescence remains unsettled and theories those included hotspot, bremsstrahlung radiation, collision-induced radiation and corona discharges, nonclassical light, proton tunneling, electrodynamic jets, fractoluminescent jets, and so forth were presented [3].

As the properties of sonoluminescence which releases too large an amount of energy and releases the energy on too short a time scale are consistent with the vacuum energy explanation, Schwinger proposed a physical mechanism based on the instantaneous collapse of a bubble for sonoluminescence in terms of photon production due to changes of quantum electrodynamic energy contained in a collapsing dielectric bubble [4].

According to the Schwinger's theory, the surface of a bubble is supposed to act as the Casimir force plates and an abrupt change of electromagnetic energy is emitted as visible light in sonoluminescent flashes when the bubble collapses. But there are some critics for the Schwinger's proposal that his estimate of the Casimir energy involved 
is inaccurate and several papers were presented to consider the missing terms [5-8]. One of them was a proposition given by Claudia Eberlein $[9,10]$ that sonoluminescence could be explained in terms of quantum vacuum radiation by moving interfaces between media of different polarization, which might be alike to the Unruh effect. Instead of their conventional quantum electrodynamic (QED) ideas for the explanation of sonoluminescence, the author tries to present another component of sonoluminescense, which is due to the Cherenkov radiation from tachyon pairs created from the zero-point fluctuations of electromagnetic field (ZPF field) contained in a collapsing bubble.

\section{THEORETICAL ANALYSIS FROM THE STANDPOINT OF ZPF THEORY}

\subsection{ZPF Radiation Due to Casimir Energy}

As shown in Figure 1, the vacuum constitutes an extremely energetic physical state.

The premier example for considering the possibility of extracting energy from the vacuum has already appeared in a paper by R.L.Forward by applying the Casimir effect [11]. Schwinger wrote papers wherein the Casimir energy released in the collapse of a spherically symmetric bubble or a cavity with a volume $V$ in a dielectric fluid can be given by [4].

$$
\begin{aligned}
& E_{\text {cavity }}=2 V \int_{0}^{K} \frac{4 \pi k^{2} d k}{(2 \pi)^{3}} \frac{\hbar c k}{2}\left(\frac{1}{\sqrt{\varepsilon_{\text {in }}}}-\frac{1}{\sqrt{\varepsilon_{\text {out }}}}\right)+\cdots \\
& =\frac{1}{6 \pi} \hbar c R^{3} K^{4}\left(\frac{1}{\sqrt{\varepsilon_{\text {in }}}}-\frac{1}{\sqrt{\varepsilon_{\text {out }}}}\right)+\cdots
\end{aligned}
$$

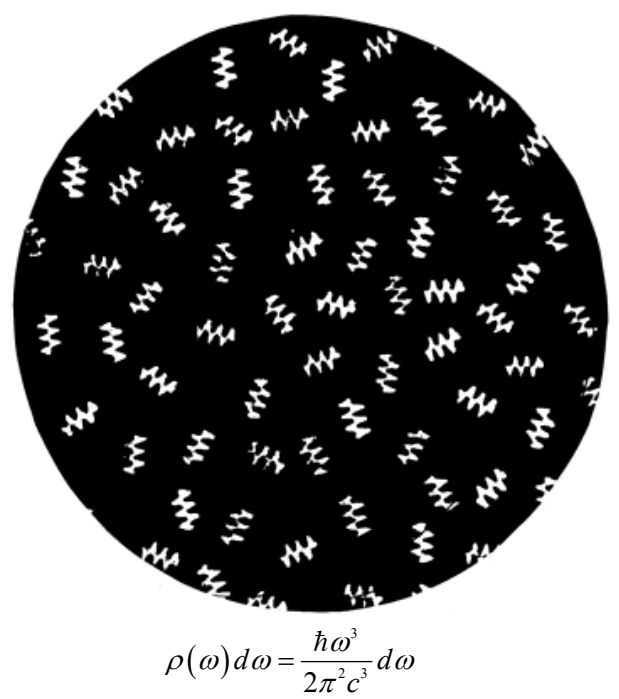

Figure 1. Zero point energy which fills the space. where $V$ is the volume of a bubble, $R$ is its radius, $K$ is a high wavenumber cutoff that characterizes the wavenimber at which the dielectric constants drop to their vacuum values, $\hbar$ is a Plank's constant divided by $2 \pi$, $c$ is a light speed and $\varepsilon_{\text {in }}$ and $\varepsilon_{\text {out }}$ are dielectric constants for outside the bubble and inside it respectively. However the possible relevance of the Casimir effects to sonoluminescence was considered to be controversy because the corresponding Casimir energy was only $E_{c} \sim 10^{-22} J$ for a rapidly collapsing bubble [12], which was about 10 ordered of magnitude too small to be relevant to sonoluminescence from the experimental results.

Instead of Schwinger's idea to explain the sonoluminescense, Liberati, Visser, Belgiorno and Sciama derived the equation in the framework of the dynamical Casimir effect to give reasonable agreement with observations, which included the contribution of dynamical Casimir effect shown as [13].

$$
\begin{aligned}
E & =\frac{1}{8 \pi n^{2} n^{\prime}}\left(\frac{n-n^{\prime}}{n n^{\prime}}\right)^{2} \hbar c K(R K)^{3} \\
& \approx \frac{1}{8 \pi n^{2}}\left(1-\frac{1}{n}\right)^{2} \hbar c R^{3} K^{4}
\end{aligned}
$$

where $n$ is a refractive index of liquid and $n^{\prime}$ is a refractive index of gas inside the bubble. However this mechanism can not explain impossibly short time scales of $\mathrm{SL}$ radiation because it is required that the overall collapse time of a bubble is $10^{-4}$ sec instead of the impossible short time scale on the order of $10^{-15} \mathrm{sec}[14]$.

Thus I have proposed another missing component of sonolumenescence due to Cherenkov radiation from tachyon pairs generated from the ZPF field contained in a collapsing bubble instead of a conventional theory for explaining sonolumenescence as a QED vacuum effect, given as follows.

\subsection{Cherenkov Radiation from Tachyon Pairs Generated in a ZPF Field in a Space}

The author proposed the possibility in his paper that the Cherenkov radiation can be generated from tachyon pairs created in a collapsing bubble shown as follows; From the wave equation taking account of the special relativity (i.e. Klein-Gordon equation) given by

$$
i \hbar \frac{\partial \psi}{\partial t}=H \psi
$$

where $H=\sqrt{p^{2} c^{2}+M^{2} c^{4}} \quad(p:$ momentum of the particle, $M$ : effective mass) and $\psi$ is a wave function for the particle, the following equation can be obtained for the accelerated particle [15]; 


$$
\frac{\partial \psi}{\partial p}=-\frac{i}{M a \hbar} \sqrt{p^{2} c^{2}+M^{2} c^{4}} \psi
$$

where $a$ is a proper acceleration of the virtual elementary particle generated from a ZPF field. If the photon is generated in a quantum region, which size is $l$, the proper acceleration of the photon becomes

$$
a=\frac{1}{m} \frac{\Delta p}{\Delta t} \approx \frac{c^{2}}{l},
$$

from the uncertainty of momentum and energy given by $\Delta p \cdot l \approx \hbar$ and $\Delta E=\Delta p \cdot c$, respectively, when we let $\Delta E=m c^{2}$ and $\Delta t=l / c$.

Then the wave function for the accelerated particle becomes [16]

$$
\begin{aligned}
& (v<c) \\
& \psi=C \cdot \exp \left[-i \frac{\omega}{2 c} l \sqrt{1-\beta^{2}}\right. \\
& \left.\times\left(\frac{\beta}{1-\beta^{2}}+\log (\hbar \omega / c)+\log (1+\beta)\right)\right] \\
& (v>c) \\
& \psi^{*}=C \cdot \exp \left[-\frac{\omega}{2 c} l \sqrt{\beta^{2}-1}\right. \\
& \left.\times\left(\frac{\beta}{\beta^{2}-1}-\log (\hbar \omega / c)-\log (1+\beta)\right)\right]
\end{aligned}
$$

From these equations, it can be seen that the wave function continues beyond the light barrier and we can see that there is a possible existence of virtual superluminal particles created from the ZPF field.

According to the WKB approximation, the penetration probability through the light barrier of the tunneling photon can be estimated by

$$
\begin{aligned}
& T_{p} \approx\left|\psi_{*}\right|^{2} /|\psi|^{2} \\
& =\exp \left[-\frac{\omega}{c} l \sqrt{\beta^{2}-1}\left(\frac{\beta}{\beta^{2}-1}-\log (\hbar \omega / c)-\log (1+\beta)\right)\right]
\end{aligned}
$$

By applying the uncertainty principle to the virtual superluminal particle, we obtain $\beta \approx 2$ [17] and then Eq.7 becomes

$$
T_{p} \approx \exp \left[-\frac{\omega}{c} l \sqrt{3}\left(\frac{2}{3}-\log (\hbar \omega / c)-\log 3\right)\right] .
$$

Supposing that $l$ has a size of the Plank length, then Eq.8 can be approximated by

$$
T_{p}(\omega) \approx \exp \left[-\gamma \cdot l_{p} \omega\right]
$$

where $l_{p}$ is a Plank length and

$$
\gamma=\frac{3 \log 3-2+3 \log (\hbar / c)}{\sqrt{3} c} \approx 5.62 \times 10^{-7} .
$$

If tachyon pairs created from the ZPF background have an electric charge, it radiates photons at the angle of $\theta_{c}=\cos ^{-1}(1 / \beta n)$, where $\theta_{c}$ is half-angle of the Chrenkov radiation from the particle moving at the speed of $\beta=v_{*} / c$ and $n$ is the index of refraction which equals to unity in a vacuum.

As the radiation field by the Cherenkov effect can be regarded as a thermal equilibrium system filled with non-radiating electromagnetic waves, it is permitted that small fraction of energy from non-radiating electromagnetic field can be radiated as blackbody radiation according to the SED theory shown as follows;

$$
\begin{aligned}
\rho_{E}(\omega) & =\frac{\hbar \omega^{3}}{2 \pi c^{3}} T_{p}(\omega) \frac{\sum_{k=0}^{\infty} k e^{-k \hbar \omega / k_{B} T}}{\sum_{k=0}^{\infty} e^{-k \hbar \omega / k_{B} T}} \\
& =\frac{\hbar \omega^{3}}{2 \pi^{2} c^{3}} \exp \left(-\gamma l_{p} \omega\right)\left[\exp \left(\frac{\hbar \omega}{k_{B} T}\right)-1\right]^{-1}
\end{aligned}
$$

where $k_{B}$ is the Boltzmann constant and $T$ is the absolute temperature of radiation.

From the calculation by Liberati et al, the total number of created photons by Casimir effect becomes [13]

$$
N \approx \frac{1}{6 \pi n n^{\prime}}\left(\frac{n-n^{\prime}}{n n^{\prime}}\right)^{2}(R K)^{3}
$$

then the average energy per emitted photon can be given by

$$
\langle E\rangle \approx E / N=\frac{3}{4} \hbar c K / n .
$$

From which, the temperature inside the collapsing bubble due to the Casimir effect can be obtained from

$$
\langle E\rangle \approx \frac{3 \pi}{2} \frac{\hbar c}{n \lambda}=k_{B} T,
$$

where $\lambda$ is a wavelength of ultraviolet cutoff frequency given by $\lambda=2 \pi / K$.

By inserting this formula to Eq.11, the energy density of sonoluminesence due to the Cherenkov radiation from tachyon pairs at the temperature generated by the Casimir effect becomes

$$
\rho_{E}(\omega)=\frac{\hbar \omega^{3}}{\pi^{2} c^{3}} \exp \left(-\gamma l_{p} \omega\right) \cdot\left[\exp \left(\frac{2 n \lambda}{3 \pi c} \omega\right)-1\right]^{-1} .
$$

\section{ZPF RADIATION FROM THE COLLAPSING BUBBLE}

From the assumption that the collapsing bubble is 
black, i.e. it perfectly absorbs all wavelength of electromagnetic radiation that requires the mean free path of photons be much smaller than the size of the bubble [2], the bubble radiates all electromagnetic energy confined inside it when it shrinks to the size less than the mean free path, which can be defined by $\bar{l}=(\kappa \rho)^{-1}$, where $\kappa$ is an absorption coefficient and $\rho$ is a density of gas. Then the total energy radiated by Cherenkov radiation becomes

$$
\begin{aligned}
E_{c} & =V \int_{0}^{\infty} \rho_{E}(\omega) d \omega=\frac{4}{3} \pi\left(R_{0}^{3}-R_{\min }^{3}\right) \\
& \times \frac{\hbar}{\pi^{2} c^{3}} \int_{0}^{\infty} \omega^{3} \exp \left(-\gamma l_{p} \omega\right)\left[\exp \left(\frac{2 n \lambda}{3 \pi c} \omega\right)-1\right]^{-1} d \omega \\
& =\frac{81 \pi^{3}}{2} \frac{\hbar c}{n^{4} \lambda^{4}}\left(R_{0}^{3}-R_{\min }^{3}\right) \zeta(4,1+\alpha)
\end{aligned}
$$

where $\alpha=3 \pi l_{p} \gamma c /(2 n \lambda), \quad R_{0}$ is the radius of a bubble which is at the beginning of a rapid collapse phase, $R_{\min }$ is a radius of the bubble when it radiated electromagnetic energy, and $\zeta(m, n)$ is a Hurwitz zeta function. Inside the collapsing bubble, the temperature rises precipitously, then atoms and molecules collides with high energy particles to create a hot plasma which makes the mean free path of photons shorter.

Thus the maximum energy emitted by the Cherenkov radiation from virtual tachyon pairs in a bubble can be roughly estimated as

$$
E \approx \frac{81}{32 \pi} \frac{\hbar c}{n^{4}} R_{0}^{3} K^{4} \zeta(4,1+\alpha),
$$

which is due to the thermal radiation of electromagnetic waves created by the Casimir effect for the collapsing bubble.

From the uncertainty principle, the path of FTL photons created from the ZPF of electromagnetic field can be estimated by $l \approx c / \omega$, from which we have $l \approx 0.4 \sim 0.8 \mu \mathrm{m}$ for the visible spectrum of the light.

Assuming that the path of the FTL photon almost equals to the free pass of photons generated inside the bubble, it is considered that energy confined in a collapsing bubble is radiated when it reaches the size less than one micron, which can explain the addition of small amount of noble gas increases the intensity of emitted light because the free pass of photons becomes shorter by the formation of hot plasma, that almost coincides with the experimental result. This mechanism of creating photons can also explain the short time scale of the radiation from the bubble because the heat can no longer escape from the bubble until it reaches the size of the path of FTL photons.

Figure 2 shows the process of a collapsing bubble, which consists of phases such as; 1) slow expansion, 2) turnaround at maximum radius, 3 ) collapse with moderate speed, 4) rapid collapse that the heat can no longer escape from the bubble, and 5) emission of light. Table 1 shows the experimental results for a collapsing bubble [18-21], where $R_{\max }$ is a maximum radius of the bubble and $E$ is a energy radiated from the bubble which is calculated from $\lambda=400 \mathrm{~nm}$.

From Eq.17, the energy radiated from the collapsing bubble can be estimated shown as a real line in Figure 3, when we set $K=2 \pi / 400(\mathrm{~nm})$, which corresponds to the upper wavelength of the light spectrum.

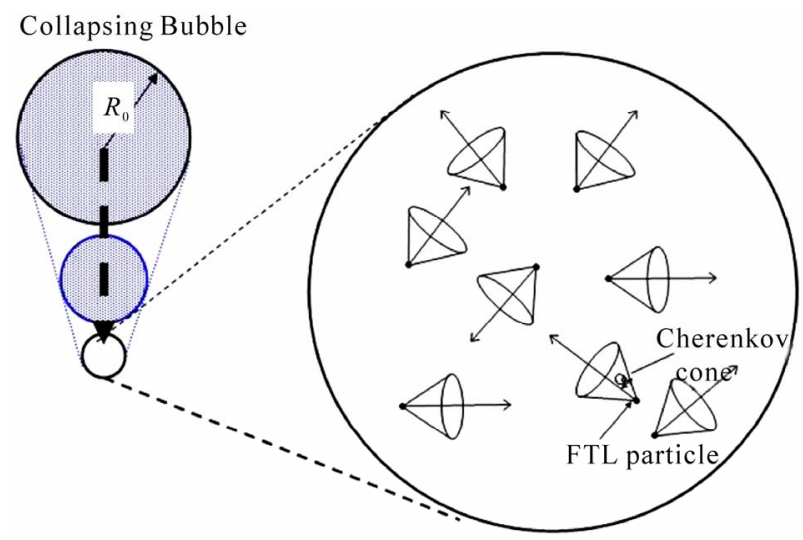

Figure 2. Collapsing bubble and Cherenkov radiation.

Table 1. Energy of flash emitted from the collapsing bubble.

\begin{tabular}{ccccc}
\hline No & $R_{\max }(\mu \mathrm{m})$ & $\begin{array}{c}\text { Number of } \\
\text { photons }\end{array}$ & $E(J)$ & Ref. \\
\hline 1 & 40 & $1 \times 10^{6}$ & $5 \times 10^{-13}$ & {$[18]$} \\
2 & 45 & - & $2.5 \times 10^{-11}$ & {$[19]$} \\
3 & 48 & $2 \times 10^{6}$ & $9.9 \times 10^{-13}$ & {$[20]$} \\
4 & 50 & $1.6 \times 10^{6}$ & $8 \times 10^{-13}$ & {$[21]$} \\
\hline
\end{tabular}

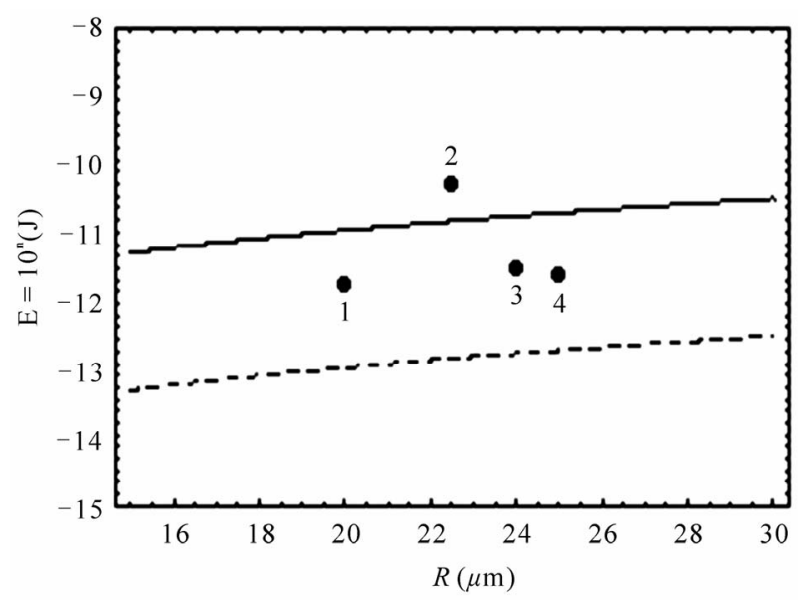

Figure 3. Radiated energy vs. radius of the bubble. 


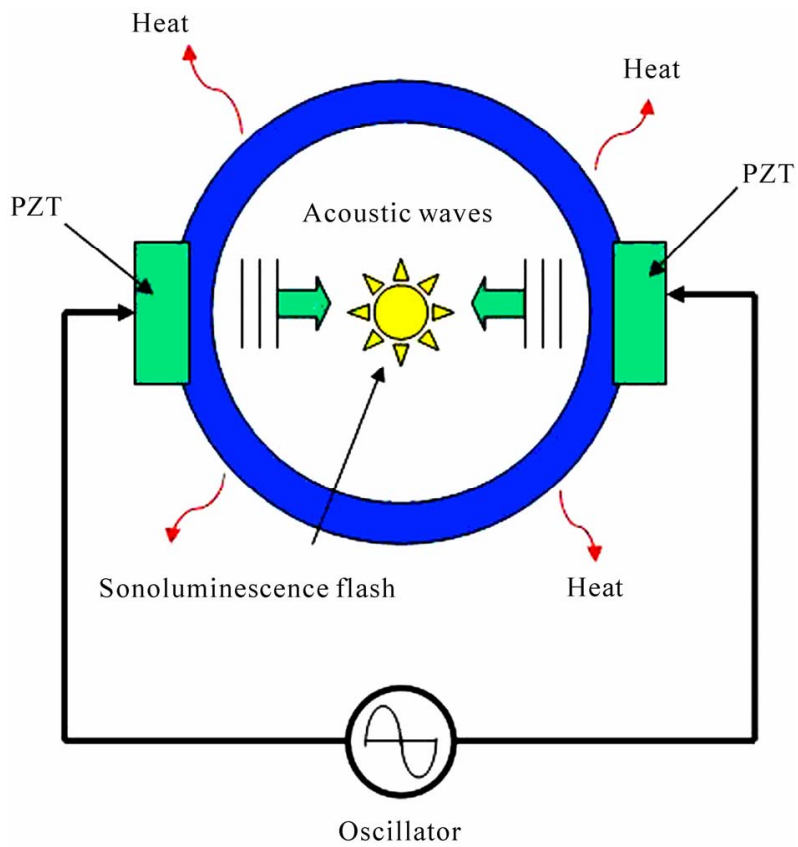

Figure 4. Schematic diagram of the fusion reactor by sonoluminescence.

The dashed line is the calculation result obtained by Eq.2 proposed by Liberati et al., which includes the contribution of dynamical Casimir effect. Assuming that $R_{0} \approx R_{\max } / 2$ [20], where $R_{0}$ is the radius of the bubble at the beginning of rapid collapse, the calculation result by Eq.17 can be shown as a solid line. In this figure, the horizontal axis is for the radius of a bubble and the vertical axis is for the emitted energy from the collapsing bubble in a log scale.

By comparing calculation results with the experimental results over plotted in this figure, we can see that energy radiation by the Cherenkov radiation becomes about 100 times the estimation by dynamical Casimir energy calculations, which almost coincides with the experimental results. Thus it can be considered that vacuum energy generated by the sonoluminescence can create heat over than $10^{7} \mathrm{~K}$, which is a temperature enough to induce a nuclear fusion by its large amount of heat during cavitation.

Thus it can be considered that the fusion temperature might be obtained by Cherenkov radiation created from ZPF field inside the collapsing bubbles by the apparatus as shown in Figure 4. R.P. Taleyarkhan and his colleagues at the Oak Ridge National Laboratory (ORNL) conducted acoustic cavitation experiments with deuterated acetone and they reported an observation of tritium and neutron output that were consistent with the occurrence of fusion at their experiment [22]. The neutron emission was also reported to be coincident with the sonoluminescence pulse, which suggests that its source was fusion caused by the sonoluminescence.

\section{COMCLUSIONS}

From the theoretical analysis, it can be seen that the Cherenkov radiation from the ZPF field in a bubble, which becomes larger than the energy radiated by the dynamical Casimir effect. By comparing them with the experimental results, it is considered that another missing component of the sonoluminescense may attribute to the Cherenkov radiation from tachyon pairs created from the ZPF field contained in a collapsing bubble, which may lead to the possible source of unlimited energy in the future.

\section{REFERENCES}

[1] Putterman, S.J. (1995) Sonoluminescence: Sound into Light. Scientific American, 272, 46-51. doi:10.1038/scientificamerican0295-46

[2] Brenner, M.P. (2002) Single-bubble sonoluminescence. Review of Modern Physics, 74, 425-484. doi:10.1103/RevModPhys.74.425

[3] Barber, B.R., Hiller, R.A., Lofstedt, R., Putterman, S.J. and Wenigner, K.R. (1997) Defining the unknown sonoluminescence. Physics Reports, 281, 65-143. doi:10.1016/S0370-1573(96)00050-6

[4] Schwinger, J. (1992) Casimir energy for dielectrics: spherical geometry, Proceedings of the National Academy of Sciences, USA, 1992, 89, 11118-11120.

[5] Carson, C.E., Molina-Paris, C., Perez-Mercader, J. and Visser, M. (1997) Schwinger's Dynamical Casimir Effect; Bulk Energy Contribution. Physics Letters B, 395, 76-81. doi:10.1016/S0370-2693(97)00009-9

[6] Carson, C.E., Molina-Paris, C., Perez-Mercader, J. and Visser, M. (1997) Casimir effect in dielectrics: Bulk Energy Contribution. Physical Review D, 56; 1262-1280. doi:10.1103/PhysRevD.56.1262

[7] Milton, K.A. and Jack Ng, Y. (1997) Casimir energy for a spherical cavity in a dielectric: Applications to sonoluminescence. Physical Review E, 55, 4207-4216.

doi:10.1103/PhysRevE.55.4207

[8] Milton, K.A. and Jack Ng, Y. (1998) Observability of the bulk Casimir effect: Can the dynamical Casimir effect be relevant to sonoluminescence? Physical Review E., 57, 5504-5510. doi:10.1103/PhysRevE.57.5504

[9] Eberlein, C. (1996) Sonoliminescence as Quantum Vacuum Radiation, Physical Review Letters, 76, 3842-3845. doi:10.1103/PhysRevLett.76.3842

[10] Eberlein, C. (1996) Theory of quantum radiation observed as sonoluminescence. Physical Review A, 53, 2772-2787. doi:10.1103/PhysRevA.53.2772

[11] Forward, R.L. (1984) Extracting electrical energy from the vacuum by cohension of charged foliated conductors. Physics Review B, 30, 1700-1702. doi:10.1103/PhysRevB.30.1700

[12] Brevik, I., Marachevsky, V.N. and Milton, K.A. (1999) Identify of the van der Waals Force and the Casimir Effect and the Irrelevance of These Phenomena to Sonoluminescence. Physical Review Letters, 82, 3948-3951. 
doi:10.1103/PhysRevLett.82.3948

[13] Liberati, S., Visser, M., Belgiornoss, F. and Sciama, D.W. (2000) Sonoluminescence as a QED vacuum effect: Probing Schwinger's proposal. Journal of Physics A: Mathematical and General, 33, 2251-2272. doi:10.1088/0305-4470/33/11/307

[14] Milton, K.A. (2000) Dimensional and dynamical aspects of the Casimir effect: Understanding the reality and significance of vacuum energy. http://arXiv.org/abs/hep-th/009173

[15] Musha, T. (2005) Superluminal effect for quantum computation that utilizes tunneling photons. Physics Essays, 18, 525-529. doi: $10.4006 / 1.3025765$

[16] Musha, T. (2009) Thermal radiation generated inside the Sun due to the cherenkov radiation from ZPF field. Far East Journal of Applied Mathematics, 37, 229-235.

[17] Musha, T. (2001) Cherenkov radiation from faster-thanlight photons created in a ZPF background. Journal of Theoretics, 3, 1-7.
[18] Milton, K.A. (1998) Sonoluminescence and the dynamical Casimir effect. The Forth Workshop on Quantum Theory under the Influence of External Conditions, Leipig, 14-18.

[19] Liberati, S., Visser, M., Belgiorus, F. and Sciama, D.W. (1999) Sonoliminescence and the QED vacuum. Proceedings of the Fourth Workshop on Quantum Field Theory under the Influence of External Conditions, World Scientific, Singapore, 1999.

[20] Putterman, S.J. and Weninger, K.R., (2000) Sonoluminescence: How bubbles turn sound into light. The Annual Review of Fluid Mechanics, 32, 445-476.

[21] Hammer, D. and Frommhold, L. (2000) Spectra of sonoluminescent rare-gas bubbles. Physical Review Letters, 85, 1326-1329.

[22] Taleyarkhan. R.P., West, C.D., Cho, J.S., Lahey Jr., R.T., Nigmatulin, R.I. and Block, R.C. (2002) Evidence for nuclear emissions during acoustic cavitation. Science, 295, 1868-1873. 